\title{
Electron Microscope Study of Sigma Phase Precipitation in an Iron-Chromium-Nickel Alloy*
}

\author{
By Soji Nenno**t, Masayoshi Tagaya**, Koji Hosomi** \\ and Zenji Nishiyama***
}

\begin{abstract}
An $\mathrm{Fe}-25 \% \mathrm{Cr}-20 \% \mathrm{Ni}$ alloy was cold-rolled up to about $20 \%$ and then annealed at $700^{\circ} \mathrm{C}$ for various periods of time up to $20 \mathrm{hr}$ in order to precipitate the sigma phase. Electron microscope observation was made on thin foils prepared by electropolishing. Deformation twins and stacking faults were found in the cold-rolled specimens. The sigma phase precipitates preferentially at grain boundaries, stacking faults and twins, and possibly at dislocation crowded regions. Precipitates of the sigma phase were observed more often at both ends of the stacking fault than at the intermediate position and the sigma phase is thought to be nucleated on the stacking fault. The nucleation of the sigma phase also seems to occur on the twin interface. But when the sigma phase precipitates at the intersection of two twins of different orientations, the sigma phase seems to be nucleated on neither of the intersecting twin planes, but on the other $\{111\}$ type plane. In most cases, the orientation relationship between the two phases, the matrix and the sigma phase, was the same as that determined by $\mathrm{X}$-ray diffraction, though there is some indication of possible deviation.
\end{abstract}

(Received July 4, 1963)

\section{Introduction}

It is well known that the sigma phase occurs in a number of binary ${ }^{(1) \sim(6)}$ and ternary(7)(8) alloy systems consisting of transition metal elements and it precipitates from the matrix of face-centered cubic, bodycentered cubic, or hexagonal close-packed structures under a suitable condition (proper composition and heat teatment). The crystal structure of the sigma phase has been determined for a few binary alloys such as $\mathrm{Fe}-\mathrm{Cr}^{(9)(10)}, \mathrm{Fe}-\mathrm{Mo}^{(9)}, \mathrm{Co}^{-\mathrm{Cr}^{(10)}(11)}, \mathrm{Ni}-\mathrm{V}^{(12)}$ and $\mathrm{Nb}-$ $\mathrm{Al}^{(5)}$. The unit cell contains 30 atoms and the space group is $\mathrm{P} 4 / \mathrm{mnm}$.

In an attempt to understand the mechanism of the sigma formation from a matrix, an Fe-25\% Cr- $20 \%$ $\mathrm{Ni}$ alloy was selected for the following reasons: (1) This alloy is of the homogeneous gamma (f.c.c.) phase when annealed at a homogenizing temperature and then furnace cooled, (2) the sigma phase precipitates from the gamma matrix when annealed at a sigma forming temperature, e.g. at $815^{\circ} \mathrm{C},(3)$ the orientation

* This paper was presented at the 1961 Spring Meeting of The Japan Institute of Metals.

** Department of Metallurgy, Faculty of Engineering, Osaka University, Osaka.

*** Institute of Scientific and Industrial Research, Osaka University, Sakai, Osaka.

† Now at Materials Science, College of Engineering, Marquette University, Milwaukee, Wisconsin, U. S. A.

(1) A. H. Sully: J. Inst. Metals, 80 (1951), 173.

(2) P. Greenfield and P. A. Beck: Trans. AIME, 206 (1956), 265.

(3) A. G. Knapton: J. Inst. Metals, 87 (1958), 28

(4) C. R. McKinsey and G. M. Faulring: Acta Cryst., 14 (1961), 701.

(5) P. J. Brown and J. B. Forsyth:" Acta Cryst., 14 (1961), 362.

(6) K. P. Gupta: Trans. AIME, 221 (1961), 1047.

(7) S. Rideout, W. D. Manly, E. L. Kamen, B. S. Lement and P. A. Beck: Trans. AIME, 191 (1951), 872.

(8) J. B. Darby and P. A. Beck: Trans. AIME, 209 (1957), 69.

(9) B. G. Bergman and D. P. Shoemaker: J. Chem. Phys., 19 (1951), 515 ; Acta Cryst., 7 (1954), 857.

(10) G..J. Dickins, A. M. B. Douglas and W. H. Taylor: J. Iron Steel Inst., 167 (1951), 27.

(11) J. S. Kasper, B. F. Decker and J. R. Belanger: J. Appl. Phys., 22 (1951), 361.

Trans. J I M relationships between the gamma and sigma phases have been determined by means of X-ray diffraction in a recent work by the authors ${ }^{(13)}$, and (4) the orientation relationships experimentally determined suggest that the nucleation of the sigma phase may occur more easily at lattice defects such as stacking faults or twin interfaces than at other places. Guarnieri et al. ${ }^{(14)}$ reported that the formation of the sigma phase in this type of alloy is accelerated by cold work prior to sigma forming heat treatment.

In order to obtain some information as to how lattice defects contribute to the precipitation of the sigma phase, the lattice defects in specimens cold-rolled up to about $20 \%$ were examined by means of transmission electron microscopy. The cold-rolled specimens were also investigated with regard to where and how the precipitation of the sigma phase occurs when subjected to subsequent sigma forming heat treatments.

\section{Experimental Procedure}

Electrolytic iron, electrolytic chromium, electrolytic nickel and metallic silicon which were mixed at a proportion to weigh $1 \mathrm{~kg}$ were melted in an alumina crucible in a vacuum $10^{-4} \mathrm{mmHg}$ and cast into an iron mould $30 \mathrm{~mm}$ in diameter. The ingot was forged and hot-rolled to a plate $0.7 \mathrm{~mm}$ thick. The plate was annealed for $2 \mathrm{hr}$ at $1200^{\circ} \mathrm{C}$ and cold-rolled to plates $0.3 \mathrm{~mm}$ thick. These plates were annealed for $6 \mathrm{hr}$ at $1050^{\circ} \mathrm{C}$ to have a homogeneous gamma structure and then cold-rolled by 5,10 and $20 \%$. Some of these cold-rolled sheets were annealed for various periods of time up to $20 \mathrm{hr}$ at $700^{\circ} \mathrm{C}$ to precipitate the sigma phase. The chemical compositions of the alloy are as follows (in wt \%): $\mathrm{Cr}: 26.07, \mathrm{Ni}: 19.05$, Si : 2.02, C: $0.006, N: 0.011,0: 0.006, F e:$ the

(12) W. B. Pearson and J. W. Christian: Acta Cryst., 5 (1952), 157.

(13) S. Nenno, M. Tagaya and Z. Nishiyama: Trans. JIM, 3 (1962), 82.

(14) G. J. Guarnieri, J. Miller and F. J. Vawter: Trans. ASM, 42 (1950), 981. 
balance. To prepare the thin foils for transmission electron microscopy, the specimens thus heat treated were thinned by electropolishing under the following conditions :

Electrolyte : Perchloric acid $100 \mathrm{cc}$, glacial acetic acid $400 \mathrm{cc}$

Current density: $0.04 \sim 0.05 \mathrm{amp} . / \mathrm{cm}^{2}$

Voltage : $10 \sim 15$ volts

Temperature : $5^{\circ} \sim 10^{\circ} \mathrm{C}$

The electron microscope used is a Hitachi HU-11, which was operated at $100 \mathrm{kV}$.

\section{Experimental Results}

\section{As cold-worked}

In the specimen annealed for $6 \mathrm{hr}$ at $1050^{\circ} \mathrm{C}$, dislocations were scarcely found. It consisted of the homogeneous gamma phase and annealing twins. When the alloy is cold-rolled, the dislocation density increases with the degree of cold work, and the feature and distribution of the dislocations seem to be similar to other face-centered cubic metals and alloys ${ }^{(15)}$.

When the degree of cold work exceeded about $10 \%$, stacking faults and deformation twins were

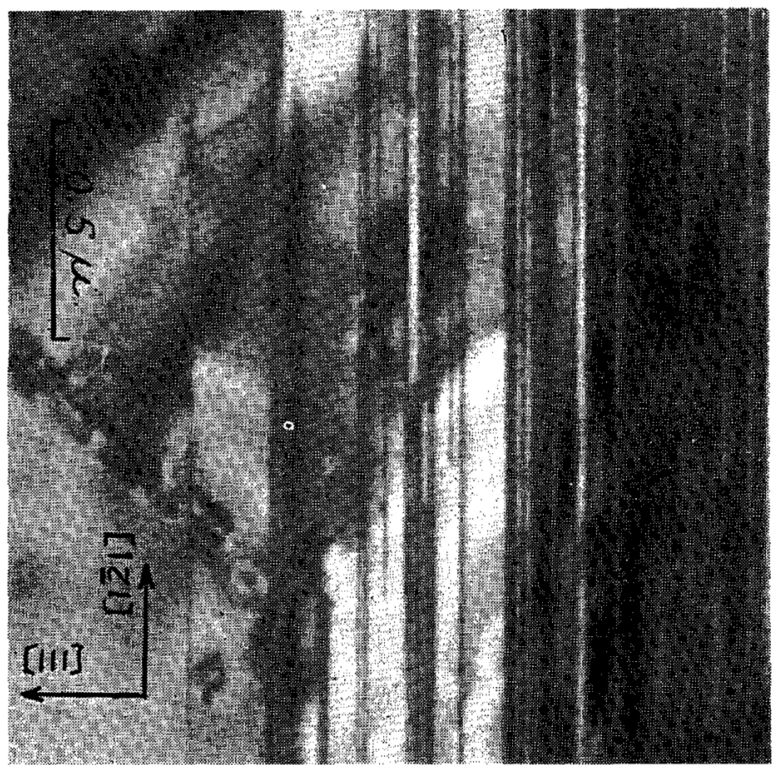

Photo. 1(a) Deformation twins; $10 \%$ cold-rolling.

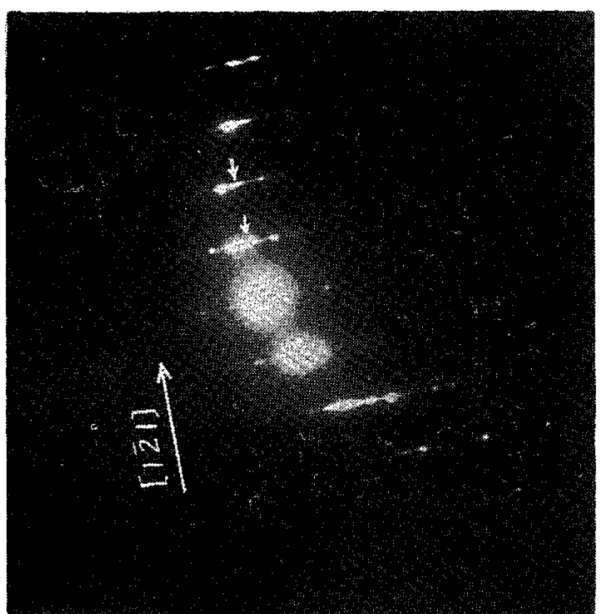

Photo. 1(b) Selected area diffraction pattern on the right hand side of Photo. 1 (a).

(15) P. B. Hirsch: J. Inst. Metals, 87 (1958/59), 406. observed. After $20 \%$ cold rolling, the deformation twins crossing each other and the cell structure of dislocations were often found. It was also found that the number of deformation twins increases with the degree of cold work. When a specimen $20 \%$ coldrolled was heated in the electron microscope up to about $550^{\circ} \mathrm{C}$, the deformation twins which were present at room temperature were found to be stable at least up to that temperature.

Photo. 1 (a) is an electron micrograph of a $10 \%$ cold-rolled specimen. From a selected area diffraction pattern on the right hand side of this field (Photo. 1 (b)), it is found that the vertical parallel bands in Photo. 1 (a) are twins which are nearly perpendicular to the foil plane. In Photo. 1 (b) streaks in the [111]r direction which are perpendicular to the twin plane may be due to the thinness of the twin plates. The central streak might be caused by the strain at the interfaces of the thin twins. Spots indicated by arrows in Photo. 1 (b) which can be interpreted neither from the matrix nor from the twin, may be attributed to junction spots or to double reflections from the matrix and the twin.

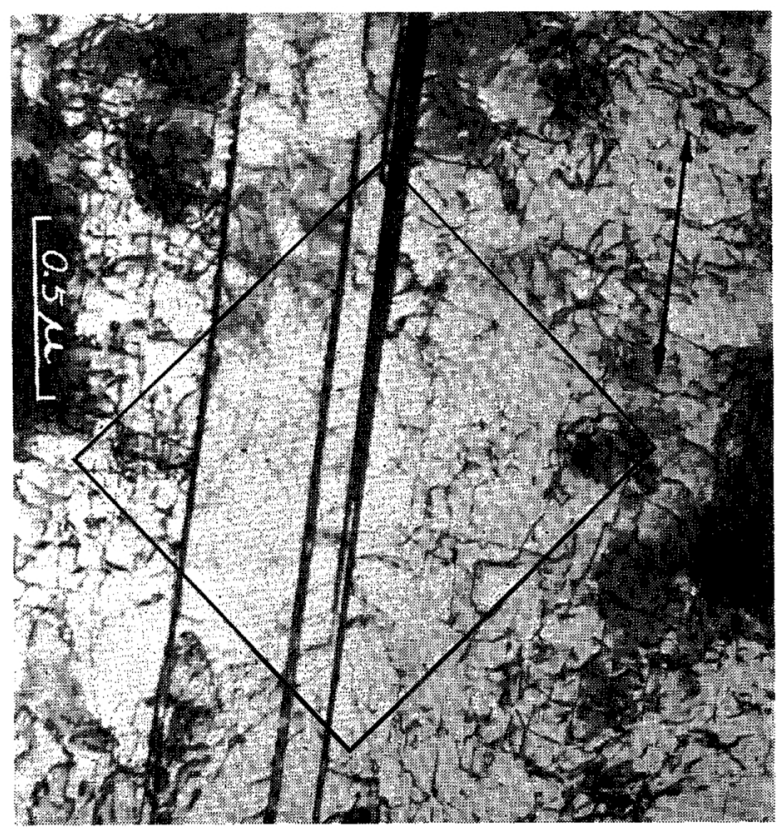

Photo. 2 (a) Twins; $3 \mathrm{hr}$ annealing at $700^{\circ} \mathrm{C}$ after $20 \%$ cold-rolling.

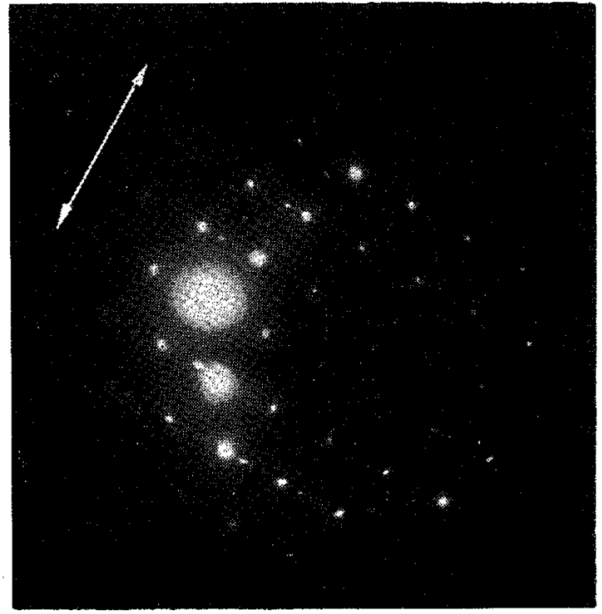

Photo. 2(b) Selected area diffraction pattern of the framed region in Photo. 2 (a). 


\section{3-hr annealing at $700^{\circ} \mathrm{C}$ after $20 \%$ cold- rolling}

In this treatment the annealing time was found to be not long enough to thoroughly precipitate the sigma phase. The precipitation occurred mostly at grain boundaries. Photo. 2 (a) shows such twins which might have been produced by cold work and grown thicker by the subsequent annealing. A diffraction pattern of the framed region in this figure is shown in Photo. 2 (b), from which it is found that the vertical bands in Photo. 2 (a) are twins perpendicular to the foil plane $(110)_{r}$.

Photo. 3 is an example of electron micrographs showing the precipitation of the sigma phase which occurred on a stacking fault A. Diffraction patterns of two regions (a) and (b) in Photo. 3 are shown in Photo. 4. (a) and (b), respectively. Photo. 4 (a) of the matrix only shows that the foil plane is roughly parallel to (110), passing through the $[1 \overline{1} 1]$ direction. Fig. 1 is an illustrative diagram for Photo. $4(\mathrm{~b})$. In analyzing this pattern, we refer to Photo. 4 (a) to pick up the matrix spots and we assume that traces $T_{1}$ and $T_{2}$ (indicated by arrows in Photo. 3 and Fig. 1) are the intersections of the foil plane with $(111)_{\gamma}$ and $(11 \overline{1})_{\gamma}$, respectively. Taking account of the obsevations that the angle between trace $T_{1}$ and $[\bar{I} I \bar{I}]_{\gamma}$ is $58^{\circ}$ and that between trace $T_{2}$ and $[\overline{1} 1 \overline{1}] \gamma$ is $32^{\circ}$, the foil plane is found to be parallel to $(2.30,3.30,1)_{\gamma}$. Sigma spots are indexed as shown in Fig. 1. Referring to this

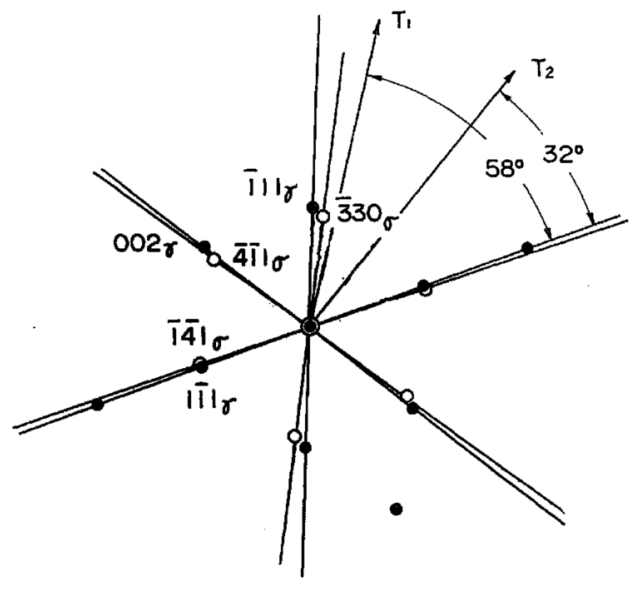

- gAMMA

- SIGMA

Fig. 1 Illustrative diagram of Photo. 4(b).

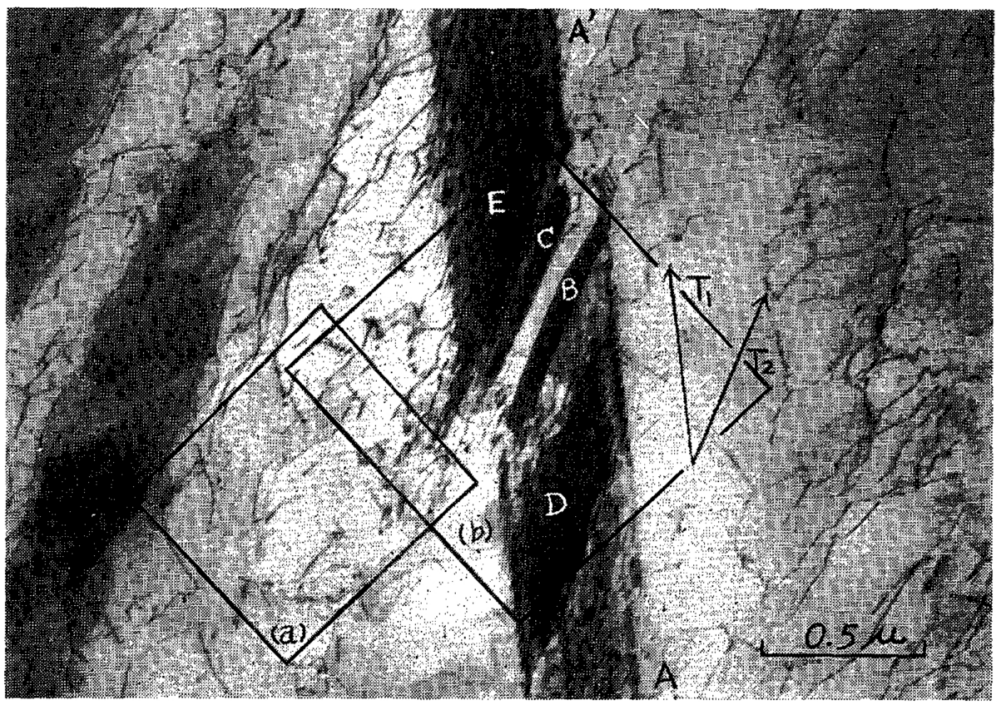

Photo. 3 Sigma phase precipitated at stacking faults; $3 \mathrm{hr}$ annealing at $700^{\circ} \mathrm{C}$ after $20 \%$ cold-rolling.

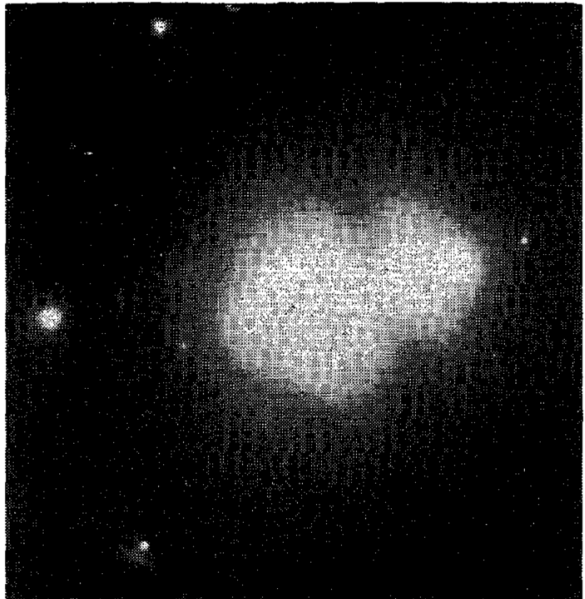

(a)

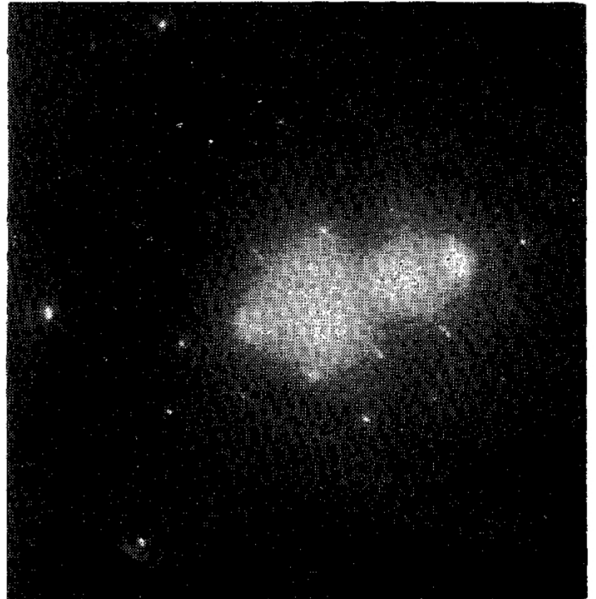

(b)

Photo. 4 Selected area diffraction patterns of the framed region (a) and (b) in Photo. 3. 
sigma phase the index of the foil normal becomes [115] $\sigma$. Using the orientation relationship $(111)_{\gamma} \|$ $(001)_{\sigma},[01 I]_{\gamma} \|[110] \sigma$ which were determined by $\mathrm{X}$-ray diffraction ${ }^{(13)}$, we convert the index of the foil normal referred to the sigma crystal axes to that referred to the gamma axes: $[115] \sigma \rightarrow[3.04,5.08,1] \gamma$, which differs from $[2.30,3.30,1] \gamma$ by only $5^{\circ}$. In the same way we have: $\left.(\bar{I} 41)_{\sigma \rightarrow(1.04,-0.818,1}\right)_{r}$ the latter is close to $(1 \overline{1} 1)_{\gamma}$ (see Fig. 1). Therfore, it is concluded that two parallel rods at $B$ and $C$ in Photo. 3 are precipitates of the sigma phase. A thickness fringe is clearly seen in the lower one, $\mathrm{B}$.

The structure observed in regions $D$ and $E$ ( An enlargement of this part is shown in Photo. 5) may be some moiré pattern ${ }^{(16)}$ due to the presence of the thin precipitate on the stacking fault plane. Thus, Photo. 3 may be interpreted in the following way: In this stacking fault. So it is thought that thin layers of the sigma phase are, at first, formed near the both ends on a stacking fault and then some part of the thin layers grow to precipitates of the sigma phase. In fact, a precipitate of the sigma phase is found at $B$ in Photo. 3 which is one end of stacking fault, A. At the other end of this stacking fault, which is far below beyond Photo. 3 and is not shown here, another precipitate of the sigma phase and a moirś pattern adjacent to it were observed.

Photo. 6 shows that precipitation of the sigma phase occurred in a row at a grain boundary.

\section{10-hr annealing at $700^{\circ} \mathrm{C}$ after $20 \%$ cold- rolling}

With this treatment, the appearance of the sigma precipitate is not much different from the one which

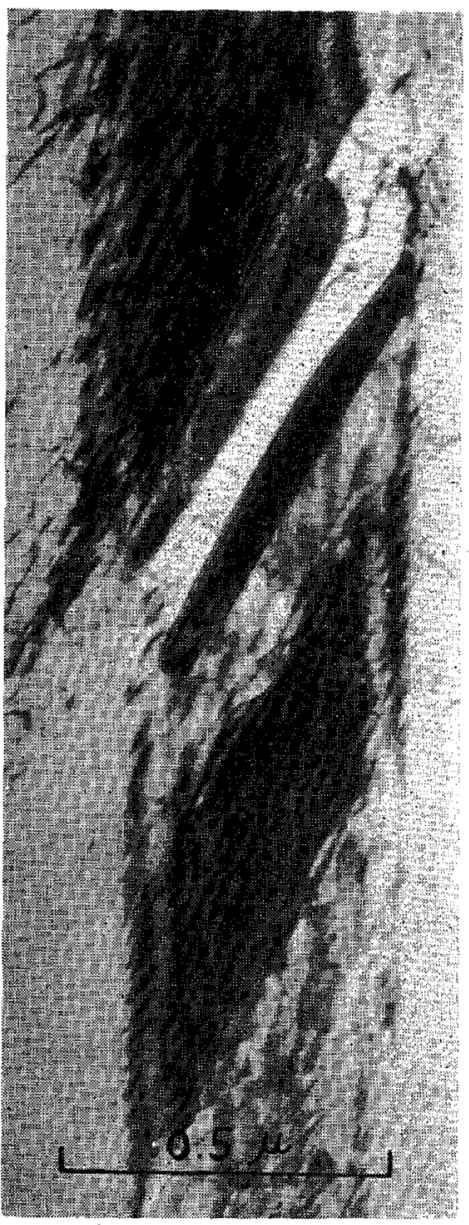

Photo. 5 Partial enlargement of Photo. 3.

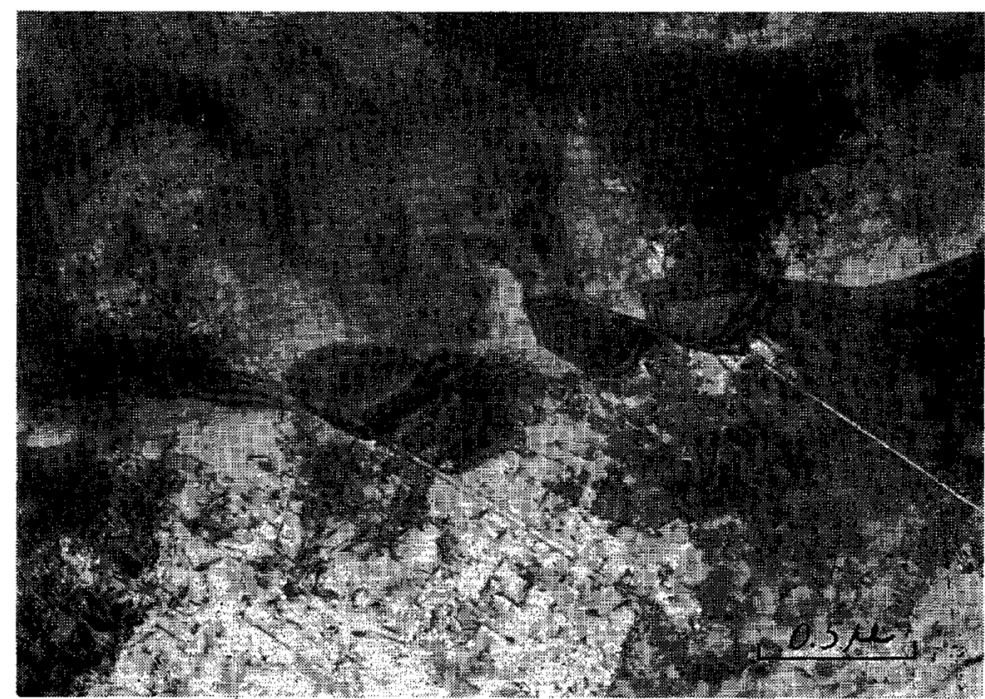

Photo. 6 Sigma phase precipitated at a grain boundary; $3 \mathrm{hr}$ annealing at $700^{\circ} \mathrm{C}$ after $20 \%$ cold-rolling.

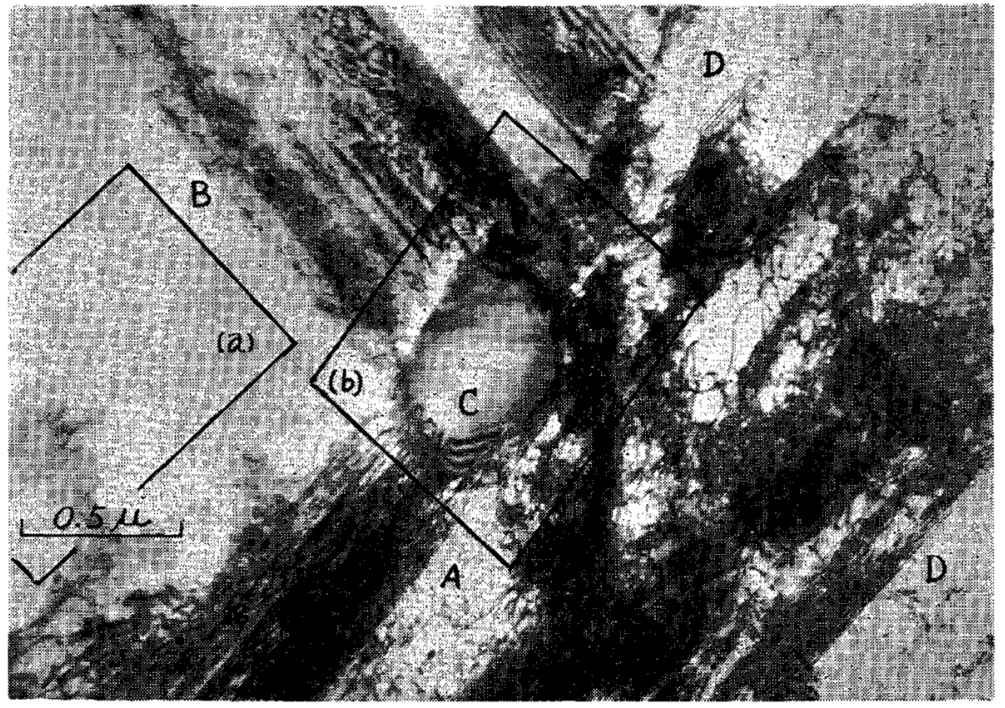

Photo. 7 Sigma phase precipitated at the intersection of two twin bands of different direction; $10 \mathrm{hr}$ annealing at $700^{\circ} \mathrm{C}$ after $20 \%$ cold-rolling. photograph, stacking faults, $\mathrm{A}$ and $\mathrm{A}^{\prime}$, are thought to lie on the different layers of $(111)_{r}$ plane. At the end of a stacking fault the sigma phase will precipitate more easily than at the intermediate place on the

(16) G.A. Bassett, J. W. Menter and D. W. Pashley : Proc. Roy. Soc., 246 A (1958), 345. was observed in the specimens annealed for $3 \mathrm{hr}$. The precipitates of sigma phase were not uniformly distributed in the grain, but were segregated in some regions.

Photo. 7 shows the sigma phase precipitated at the intersection of two twin bands of different direction. 
Photo. 8 (a) and (b) show diffraction patterns of framed areas (a) and (b) in Photo. 7, respectively. Fig. 2 is an illustrative diagram for Photo. 8 (b). The trace directions of bands, $\mathrm{A}$ and $\mathrm{B}$, in Photo. 7 are given as $T_{1}$ and $T_{2}$ in Fig. 2. From Photo. 8 (a), the index of

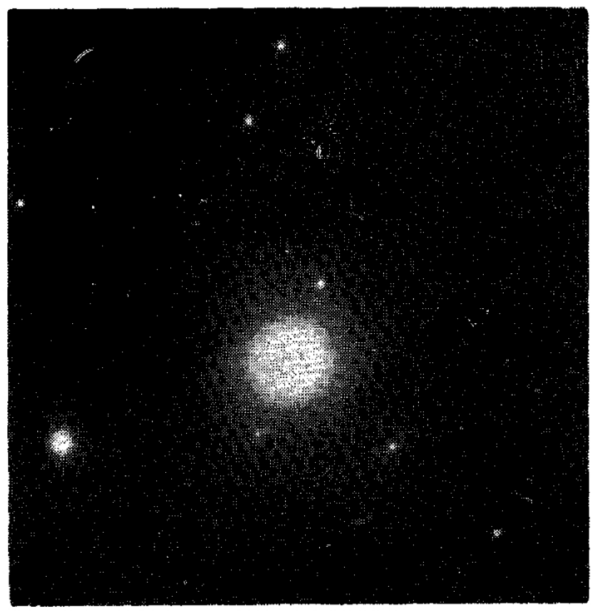

(a)

Photo. 8 Selected area diffraction patterns of the framed region (a) and (b) in Photo. 7.

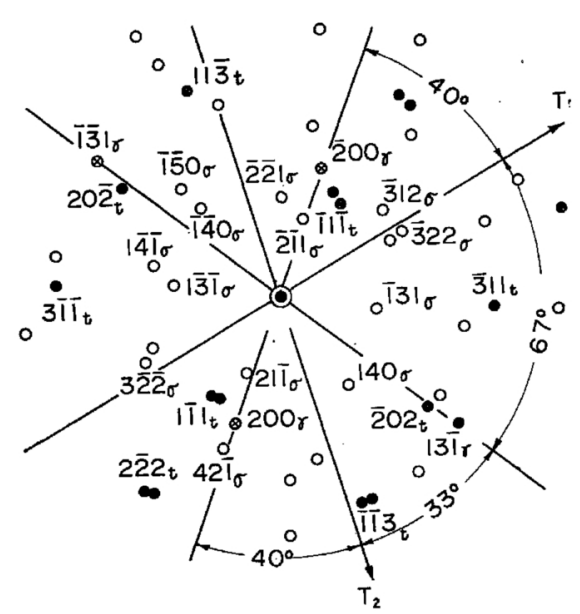

- gAMMA MATRIX

- gamma twin

- SIGMA

Fig. 2 Illustrative diagram of Photo. 8 (b).

the foil plane is found to be $(013)_{\gamma}$. Direction $T_{1}$, whose index is $[4 \overline{3} 1] \gamma$, is the intersection of two planes $(013)_{\gamma}$ and $(11 \overline{1})_{\gamma}$. Direction $T_{2}$ having an index of $[43 \bar{I}]_{\gamma}$ is the intersection of $(013)_{\gamma}$ and $(1 \overline{1} 1)_{\gamma}$. Diffraction spots denoted by a solid circle are interpreted as those from a twin formed on $(11 \overline{1})_{\gamma}$, which corresponds to the band $A$. It was also found that the bands $D$ in Photo. 7 are twins and the fringes at both sides of the twins are from the twin/matrix interfaces. The band B seems to be a twin formed on $(1 \bar{I} 1)_{\gamma}$, because the contrast of the band $B$ was reversed to some extent in a dark field image, which was taken with the reflections indicated by a white circle in Photo. 8 (b). The diffraction spots in Photo. 8 (b) which belong to neither the matrix nor the twins are indexed as the reflections from the sigma crystal (see Fig. 2). From this, we obtain the index of foil normal referred to the sigma crystal axes as [4i]7] $\sigma$.
Using the orientation relationship between the gamma and sigma phases, $(\overline{1} 11)_{\gamma}\left\|(001)_{\sigma},[110]_{\gamma}\right\|[110]_{\sigma}$, we convert the index of the foil normal: [4 17$] \sigma \rightarrow$ $[0.406,1,2.36]_{\gamma}$. The angle between $(0.406,1,2.36)_{\gamma}$ and $(013)_{\gamma}$ is $12^{\circ}$. $(200)_{\gamma}$ and $(13 \overline{1})_{\gamma}$ are deviated from

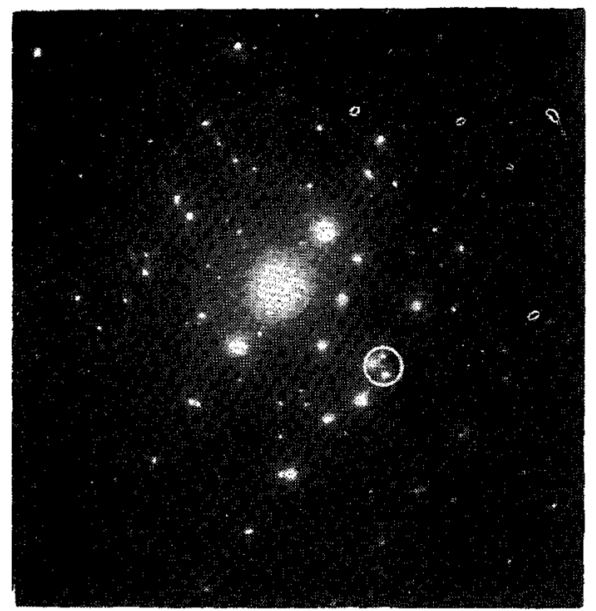

(b)

$[0.406,1,2.36]_{\gamma}$ by $9^{\circ}$ and $7^{\circ}$, respectively. The intersection of $(11 \bar{I})_{\gamma}$ and $(0.406,1,2.36)_{\gamma}$ is found to be $[5.66,-4.66,1] \gamma$ which is expected to correspond to the direction $T_{1}$. The angle between $[5.66,-4.66$, $1]_{\gamma}$ and $[13 \overline{1}]_{\gamma}$ is $68^{\circ}$ which is in good agreement with the observed one, $67^{\circ}$ (see Fig. 2). The intersection of $(1 \bar{I} 1)_{\gamma}$ and $(0.406,1,2.36)_{\gamma}$ is $[2.39,1.39$, I] $r$ which should correspond to the direction, $T_{2}$. The angle between $[2.39,1.39, \overline{1}]_{\gamma}$ and $[13 \overline{1}]_{\gamma}$ is $39^{\circ}$ which is in fairly good agreement with the observed value, $33^{\circ}$. Therefore, the precipitate $\mathrm{C}$ in Photo. 7 is considered to be the sigma phase precipitated at the intersection of two twins, A and B.

In the previous paper ${ }^{(13)}$, it was suggested that the sigma phase may more easily be nucleated at lattice defects such as stacking faults or twin interfaces than at other places. This implies that the habit plane of the sigma precipitation would be the plane of stacking fault or of twin. In the present case, the sigma precipitation did not occur on either $(11 \overline{1})_{\gamma}$ or $(1 \overline{1} 1)_{\gamma}$ twin planes, but occurred on $(\overline{1} 11)_{\gamma}$ under the relation$\operatorname{ship}(\overline{1} 11)_{\gamma}\left\|(001)_{\sigma},[110]_{\gamma}\right\|[110]_{\sigma}$. The horizontal stripes on the sigma precipitate $\mathrm{C}$ in Photo. 7 is interpreted as the intersection of $(100)_{\sigma}$ and the foil plane whose normal is $[4 \overline{1} 7] \sigma$, because the direction of this intersection is $\left[0,7(c / a)^{2}, 1\right] \sigma$. Marcinkowski et al. ${ }^{(17)}$ reported that sequence faults in the $\mathrm{Fe}-\mathrm{Cr}$ sigma phase occur on a $(100)_{\sigma}$ type plane. Since the composition of the sigma phase precipitated in the alloy used in the present work is essentially the same as that of the Fe-Cr sigma phase, the horizontal stripes on the sigma precipitate $\mathrm{C}$ are considered the image of the faults formed on $(100)_{\sigma}$ planes.

Photo. 9 is another example of the sigma phase precipitation at the intersections of two twins of different orientation. Sequence faults in the precipitate of the sigma phase are also found at $\mathrm{A}$ in Photo. 9.

(17) M. J. Marcinkowski and D. S. Miller: J. Appl. Phys., 33 (1962), 766 , 
In Photo. 10*, the faults are also clearly seen in the sigma precipitate.
Photo. 11 shows precipitation of the sigma phase which took place within the matrix. Photo. 12 (a) and

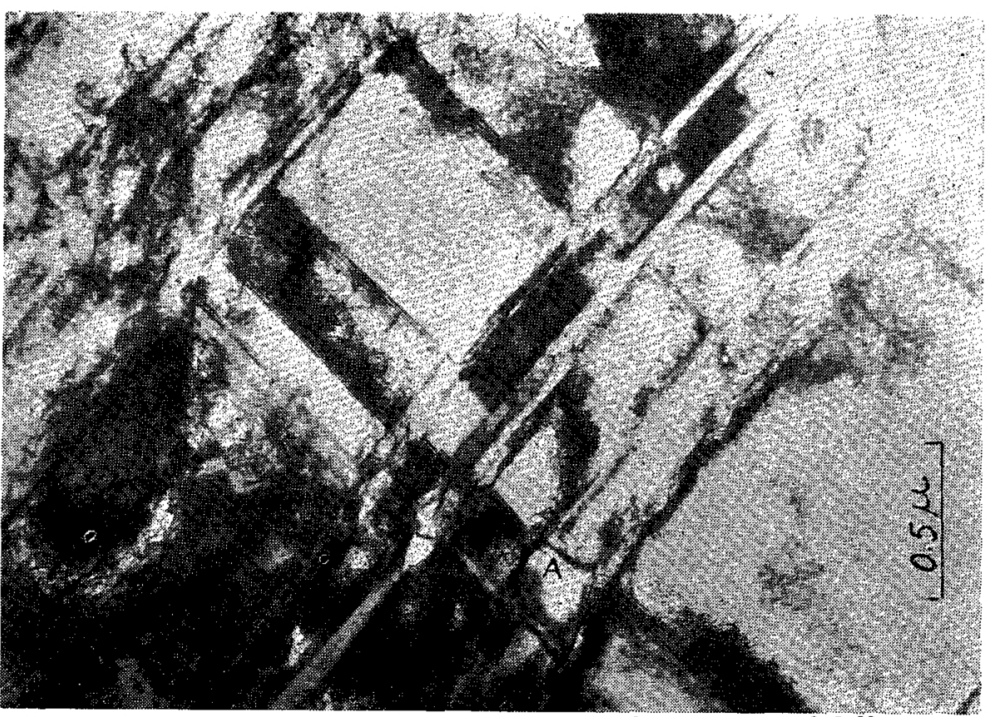

Photo. 9 Sigma phase precipitated at the intersections of two twins of different orientation; $10 \mathrm{hr}$ annealing at $700^{\circ} \mathrm{C}$ after $20 \%$ cold-rolling.

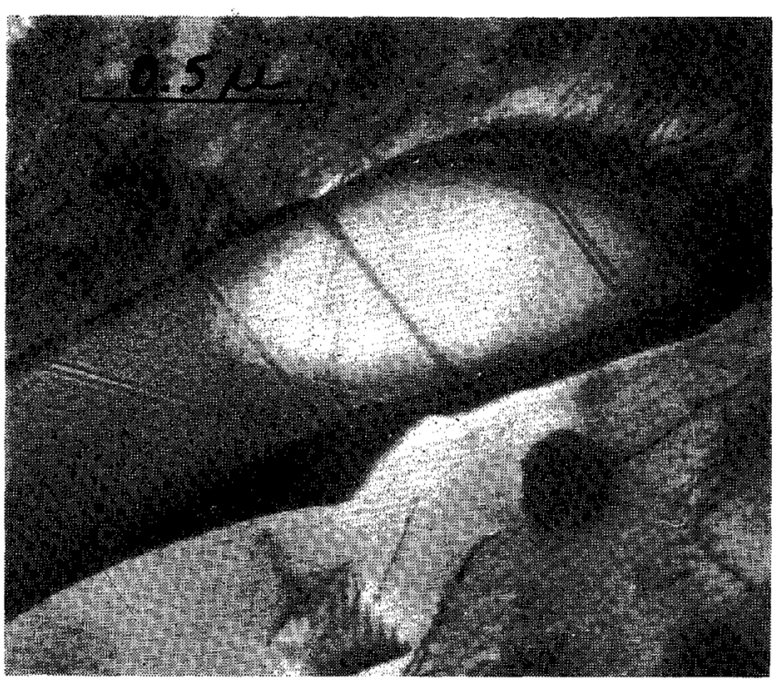

Photo. 10 Sequence faults in the well developed sigma precipitate; $3 \mathrm{hr}$ annealing at $700^{\circ} \mathrm{C}$ after cold-rolling. (b) are diffraction patterns of framed areas (a)** and (b) in Photo. 11, respectively. Fig. 3 is an illustrative diagram for Photo. 12 (b). As seen from Photo. 12 (a), the index of the foil normal is approximately [110] . From Fig. 3, it may be expressed as [4115] $\sigma$.

Using the orientation relationship $(11 \overline{1})_{\gamma} \|(001)_{\sigma}$, $[011]_{\gamma} \|[\overline{1} 10] \sigma$, we convert the index of the foil normal : $[\overline{4} 115]_{\sigma} \rightarrow[1.62,1,-0.336]_{\gamma}$ which is inclined from $[110]_{\gamma}$ by $16^{\circ} 30^{\prime}$. In the same way, we convert the indices of the sigma spots in Fig. 3 :

$$
\begin{aligned}
(\overline{1} 40)_{\sigma} \rightarrow & (-1.06,2.06,1)_{\gamma} \text { which corresponds } \\
& \text { to }(\overline{1} 11)_{\gamma}, \\
(\overline{4} 1 \overline{1})_{\sigma} \rightarrow & (0.274,0.230,2)_{\gamma} \text { which corresponds } \\
& \text { to }(002)_{\gamma}, \\
(\overline{3} \overline{3} \overline{1})_{\sigma} \rightarrow & (0.823, \bar{I}, 1)_{\gamma} \text { which corresponds to }(\overline{1} 1)_{\gamma} .
\end{aligned}
$$

Using these converted indices of the sigma spots, the angle between each reflection plane of the sigma phase and the corresponding one of the gamma phase

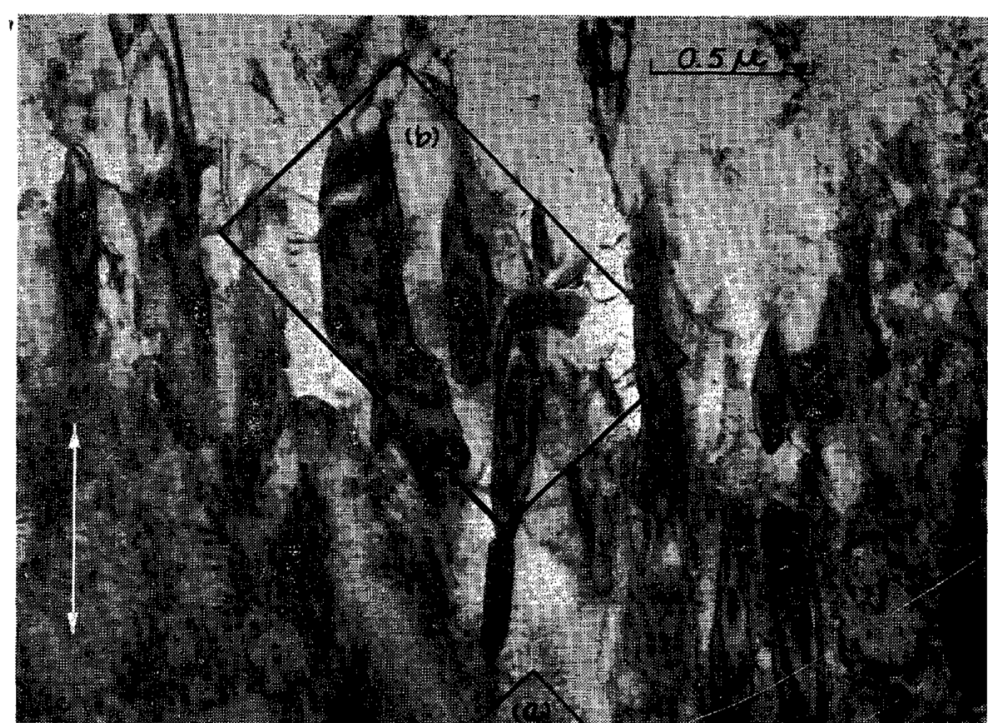

Photo. 11 Sigma phase precipitated within the matrix; $10 \mathrm{hr}$ annealing at $700^{\circ} \mathrm{C}$ after $20 \%$ cold-rolling.

* This electron micrograph was taken of a specimen annealed for $3 \mathrm{hr}$ at $700^{\circ} \mathrm{C}$ after $20 \%$ cold-rolling.
** Area (a), where there is no precipitation, has a rectangular from extending below Photo. 11. 
was calculated. The result of this calculation is listed in Table 1. From this table it is noticed that the calculated angle between $(\overline{1} 40)_{\sigma}$ and $(\overline{1} 11)_{\gamma}$ appears too large, although the orientation relationship $(11 \overline{1})_{\gamma}$ $\left\|(001)_{\sigma},[011] \gamma\right\|[\overline{1} 10]_{\sigma}$ gives a fairly satisfactory from that of the bright field image. Therefore, all these precipitates have the same orientation with respect to the matrix.

Photo. 14 is another example of the sigma precipitation which occurred within the matrix, in which the

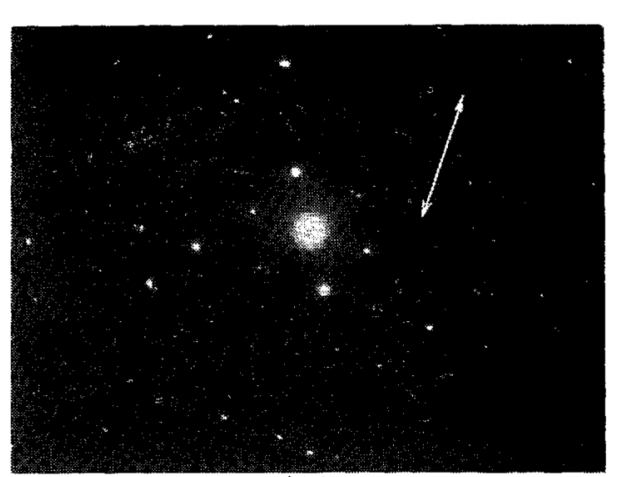

(b)

Photo. 12 Selected area diffraction patterns of area (a) (no precipitation) and (b) in Photo. 11.

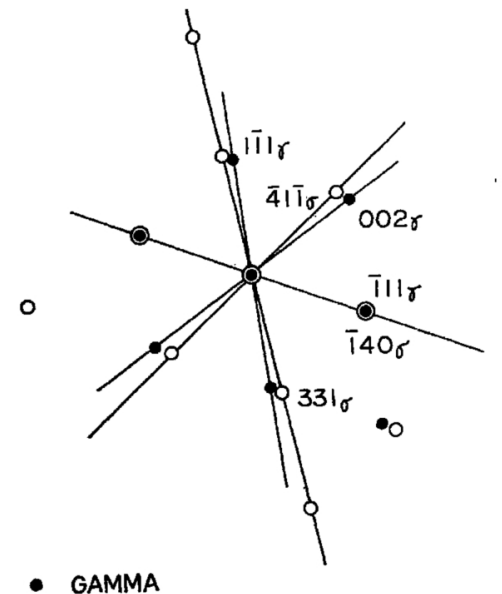

- sigma

Fig. 3 Illustrative diagram of Photo. 12 (b).

interpretation to the diffraction pattern. This is suggestive of possible deviation of the actual orientation relationship from the one used in the above calculation.
Table 1 Angles between two planes of the gamma and/or sigma phases.

\begin{tabular}{|c|c|c|c|c|}
\hline$\left(h_{1} k_{1} l_{1}\right)$ & $\left(h_{2} k_{2} l_{2}\right)$ & $\operatorname{Angle}_{(c a l c .)}$ & \multicolumn{2}{|c|}{$\begin{array}{l}\text { Angle(obs.) from } \\
\text { Photo. } 12 \text { (a) or (b) }\end{array}$} \\
\hline $\begin{array}{l}(\overline{1} 40)_{\sigma} \\
(\overline{4} \overline{1} 1) \\
(\overline{3} \overline{1} \overline{1}) \\
\sigma\end{array}$ & $\begin{array}{l}(\overline{1} 11) r \\
(002) r \\
(111) r\end{array}$ & $\begin{array}{rr}19^{\circ} & 15^{\prime} \\
9^{\circ} & 35^{\prime} \\
3^{\circ} & 35^{\prime}\end{array}$ & $12(\mathrm{a})$ & $\begin{array}{c}12(\mathrm{~b}) \\
7^{\circ} \\
4^{\circ}\end{array}$ \\
\hline$(\overline{1} 40)_{\sigma}$ & $\begin{array}{l}(\overline{4} 1 \overline{1})_{\sigma} \\
(\overline{3} \overline{3} 1)_{\sigma}\end{array}$ & $\begin{array}{l}65^{\circ} 20^{\prime} \\
53^{\circ} \quad 0^{\prime}\end{array}$ & & $\begin{array}{l}62^{\circ} \\
60^{\circ}\end{array}$ \\
\hline $\begin{array}{l}(\overline{1} 11) r \\
(002) r\end{array}$ & $\begin{array}{l}(002) r \\
(1 \overline{1} 1) r\end{array}$ & $\begin{array}{ll}54^{\circ} & 44^{\prime} \\
54^{\circ} & 44^{\prime}\end{array}$ & $\begin{array}{l}56^{\circ} \\
60^{\circ}\end{array}$ & $62^{\circ}$ \\
\hline
\end{tabular}

sigma precipitates of two different habits are found. The two have different orientations, as evidenced by a dark field image given in Photo. 15. It is thought that these sigma precipitates might have been formed on two types of $\{111\} \gamma$ planes, possibly on stacking faults, and the stacking faults might have disappeared during the growth of the precipitates.

Photo. 16 shows a recrystallized ragion around the sigma precipitates which are denoted by A. Ribbons

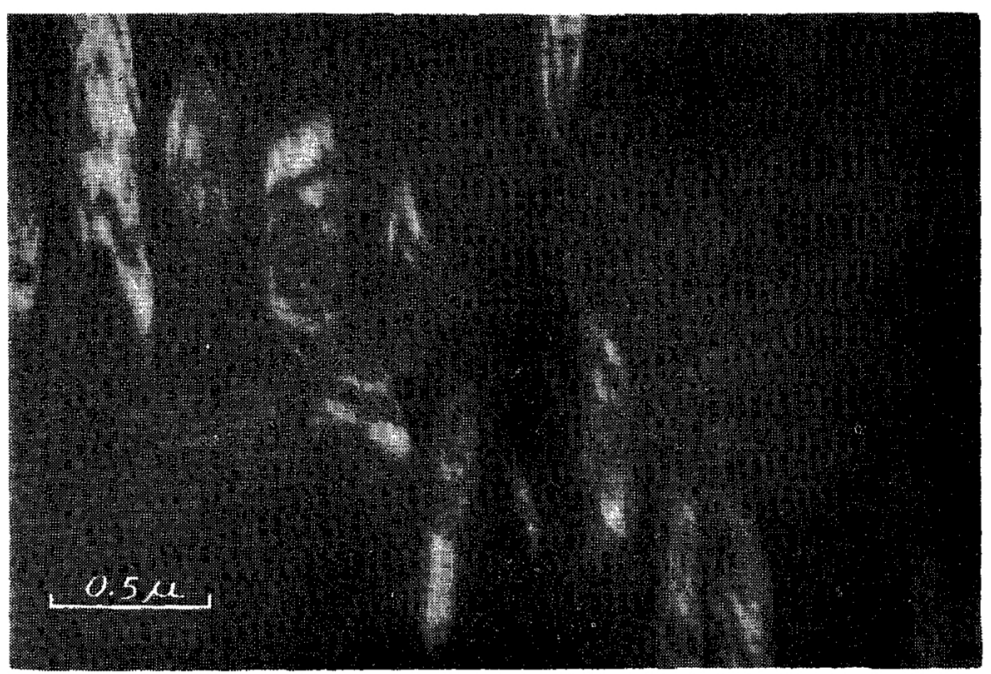

Photo. 13 A dark field image corresponding to Photo. 11.

A dark field image taken with $(331)_{\sigma}$ reflection is shown in Photo. 13. It is noticed from this figure that the contrast of each sigma precipitate is reversed at $B$ and $B^{\prime}$ seem to be twins, possibly annealing twins which might have grown from deformation twins. This reasoning is based on the finding in Photo. 2 (a) 
that deformation twins grow thicker when a cold-rolled specimen is annealed at $700^{\circ} \mathrm{C}$. A non-recrystallized region can be seen in $\mathrm{C}$ at the right bottom corner, a cold-worked $25 \% \mathrm{Cr}-20 \% \mathrm{Ni}$ stainless steel. As shown in the electron micrographs, however, the sigma precipitation occurs without recrystallization of the

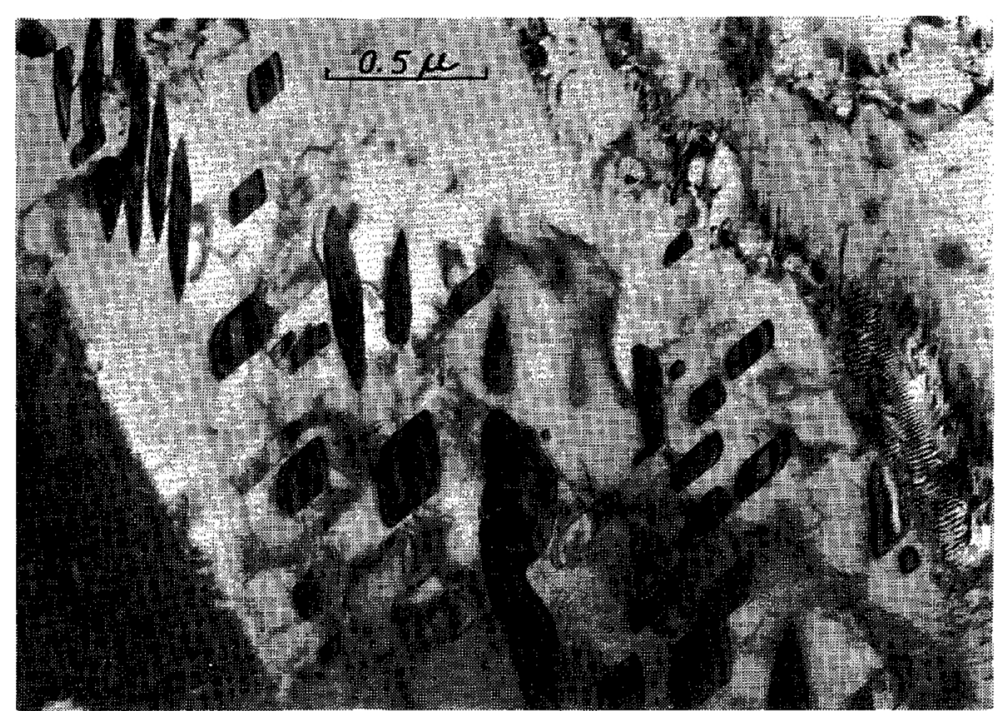

Photo. 14 Sigma precipitates of two different orientations occurred within the matrix; $10 \mathrm{hr}$ annealing at $700^{\circ} \mathrm{C}$ after $20 \%$ cold-rolling.

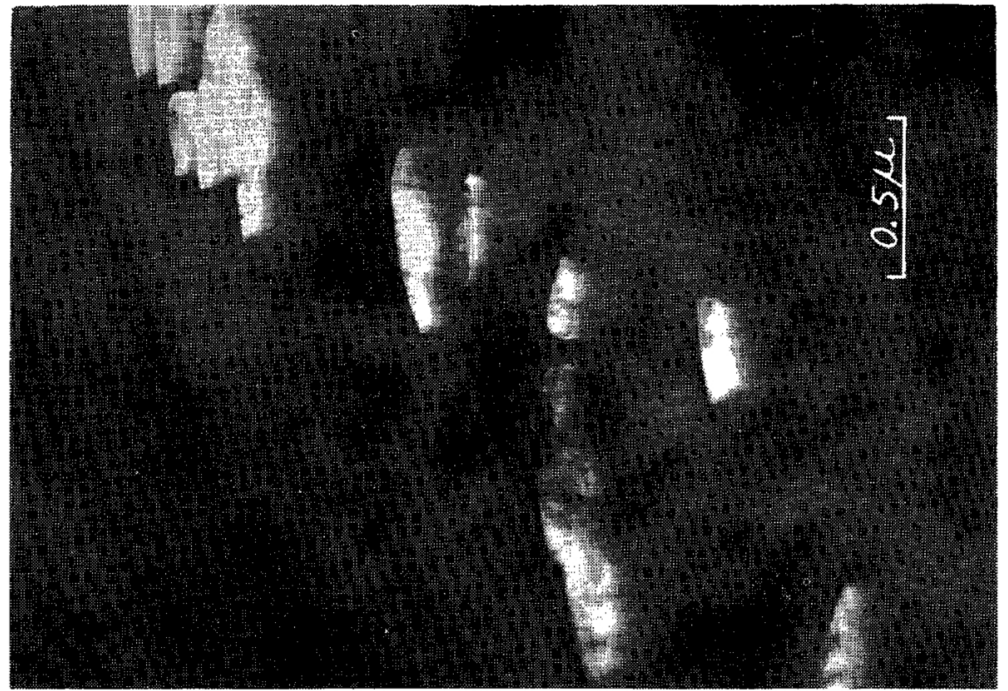

Photo. 15 A dark field image corresponding to Photo. 14 .

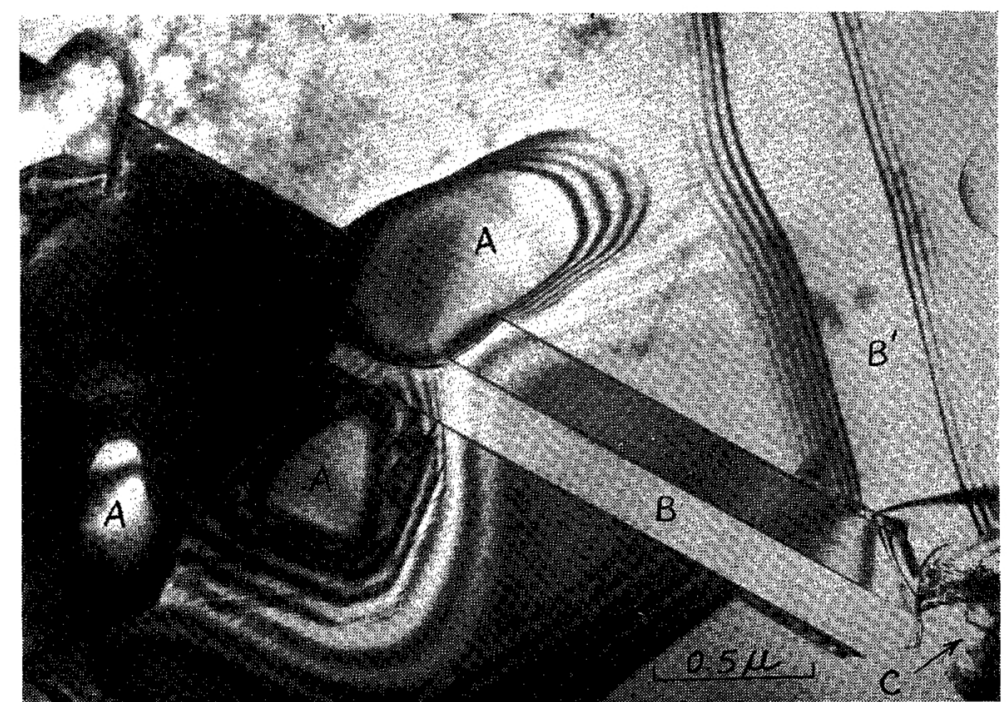

Photo. 16 A recrystallized region around the sigma precipitates; $10 \mathrm{hr}$ annealing at $700^{\circ} \mathrm{C}$ after $20 \%$ cold-rolling.

Lena et al. ${ }^{(18)}$ have proposed that recrystallization is essential for acceleration of sigma phase formation in

(18) A. J. Lena and W. E. Curry: Trans. ASM, 47 (1955), 193. 
matrix. In Photo. 10, there is a region close to the sigma precipitate where dislocations are quite few. Hence, recovery seems to occur near precipitates of the well-grown sigma phase.

\section{20 -hr annealing at $700^{\circ} \mathrm{C}$ after $10 \%$ cold- rolling}

In specimens which received this treatment, the dislocation density is comparatively low. Sigma precipitates are sparse in distribution, occurring mainly at grain boundaries, twin boundaries and in the matrix crowded with dislocations.

Photo. 17 shows that the sigma phase precipitates at a twin boundary. From the selected area diffraction

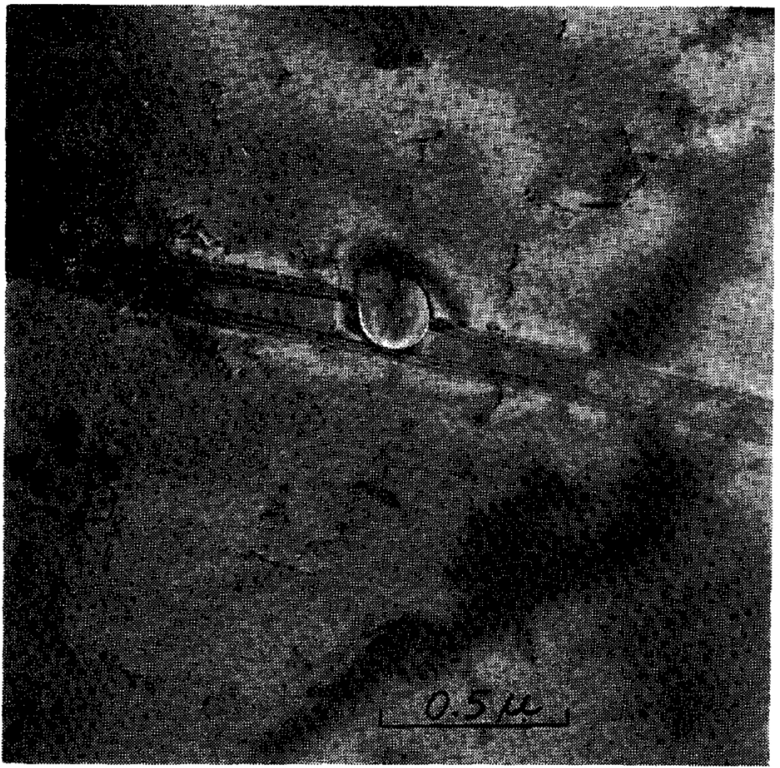

Photo. 17 Sigma phase precipitated at a twin boundary; $20 \mathrm{hr}$ annealing at $700^{\circ} \mathrm{C}$ after $10 \%$ cold-rolling.

patterns the indices of the foil plane and the twin plane are found to be $(001)_{\gamma}$ and $(111)_{r}$, respectively, and the precipitate is identified as the sigma phase which approximately satisfies the orientation relation$\operatorname{ship}(111)_{\gamma}\left\|(001)_{\sigma},[\overline{101}]_{\gamma}\right\|[110]_{\sigma}$. The fringes at both sides of the twin in Photo. 17 correspond to the matrix/twin interfaces. It is thought that the twin was grown from deformation twins formed by coldrolling and the sigma phase was nucleated on the twin/matrix interface. This will support our suggestion made in the previous paper(13).

Photo. 18 shows an example of sigma precipitation occurring at incoherent twin boundaries at $\mathrm{A}$ and $\mathrm{B}$. In Photo. 19, a precipitate of the sigma phase is seen at $\mathrm{A}$, where dislocations are crowded.

\section{Conclusion}

(1) In this alloy deformation twins and stacking faults are formed by cold-rolling. The former are stable at least up to $550^{\circ} \mathrm{C}$ and possibly grow thicker at a temperature as high as $700^{\circ} \mathrm{C}$.

(2) The sigma phase precipitates preferentially at the grain boundary, the stacking fault and the twin, and possibly at the region crowded with dislocations.

(3) In the case of stacking fault, precipitation of the sigma phase was more often observed at both ends of the stacking fault than at its intermediate position. Therefore the sigma phase is thought to be nucleated at the half dislocation of the stacking fault.

(4) In case the sigma phase is formed at a twin, the

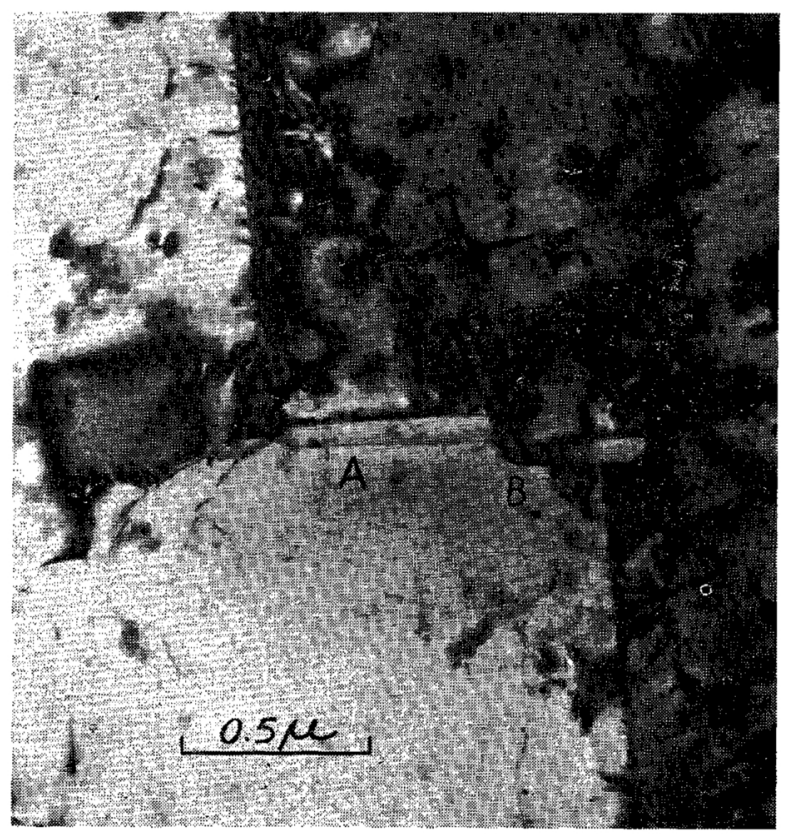

Photo. 18 Sigma phase precipitated at incoherent twin boundaries: $20 \mathrm{hr}$ annealing at $700^{\circ} \mathrm{C}$ after $10 \%$ cold-rolling.

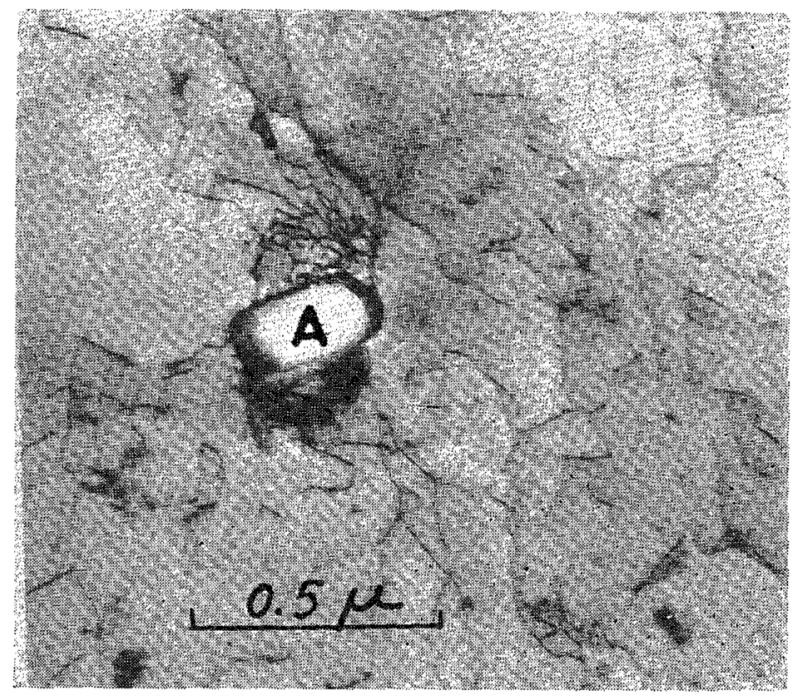

Photo. 19 A sigma precipitate seen at $\mathrm{A}$, around which dislocations are crowded; $20 \mathrm{hr}$ annealing at $700^{\circ} \mathrm{C}$ after $10 \%$ cold-rolling.

nucleation of the sigma phase is thought to occur on the twin plane. But when the sigma phase precipitates at the intersection of two twins of different orientation, the sigma phase nucleates on neither of the intersecting twin planes, but on another $\{111\}$ type plane.

(5) In most cases, the orientation relationship between the sigma and gamma phases is the same as that obtained by $\mathrm{X}$-ray diffraction, though there is some indication of possible deviation.

\section{Acknowledgment}

The authors wish to thank Mr. A. Kore'eda for his 
assistance in taking electron micrographs. They are much indebted to Dr. Y. Kojima, the Director of the Central Research Laboratory, Sumitomo Metal Industries Ltd. for chemical analysis of the alloy used in the present work. Thanks are also due to Professor
W. M. Hirthe, Marquette University for helpful discussion. This work was partially supported by the grant from the Scientific Research Fund of the Ministry of Education. 